\title{
RF Source-Seeking by a Micro Aerial Vehicle using Rotation-Based Angle of Arrival Estimates
}

\author{
Sriram Venkateswaran, Jason T. Isaacs, Kingsley Fregene, Richard Ratmansky, Brian M. Sadler, \\ João P. Hespanha and Upamanyu Madhow
}

\begin{abstract}
We investigate a computationally and memory efficient algorithm for radio frequency (RF) source-seeking with a single-wing rotating micro aerial vehicle (MAV) equipped with a directional antenna. The MAV is assumed to have no knowledge of its position and to have only an estimate of orientation through a magnetometer. A key novelty of our approach is in exploiting the rotation of the MAV and the directionality of its RF antenna to derive estimates of the angle of arrival (AOA) at each rotation. The MAV then follows the estimated direction until the next rotation is complete. We prove convergence of this greedy algorithm under rather weak assumptions on the noise associated with the AOA estimates, using recent results on the property of recurrence for systems governed by stochastic difference inclusions. These convergence results are supplemented by simulations quantifying the amount of excess travel, relative to the straight line distance to the source. Indoor experiments using Lockheed Martin's Samarai MAV demonstrate the efficacy of the greedy algorithm both for static source-seeking, and for the more challenging problem of tracking a moving source.
\end{abstract}

\section{INTRODUCTION}

We consider the problem of drawing in a micro aerial vehicle (MAV) towards an RF source by using the received signal strength (RSS) to guide the MAV's trajectory. The RF beacon could originate from a source for search and rescue in civilian/military operations, or from a sensor that wishes to establish an on-demand high data rate link with the MAV. There are two key challenges in solving this problem: first, since MAVs are very small and light, the computational power and the size of the RF sensors on-board an MAV are highly constrained. On the other hand, the spatial RF field is complex and it is difficult to make inferences from: waves scattered off reflectors lead to numerous local minima and maxima in signal strength at different locations, rendering simple source-seeking algorithms that rely on the local gradient information of the underlying field very fragile. We alleviate these difficulties by jointly designing the MAV platform, the on-board sensors, and the sourceseeking algorithm. This joint design provides us with enough flexibility to adhere to all the complexity constraints and yet

S. Venkateswaran, J. T. Isaacs, J. P. Hespanha and U. Madhow are with the Department of Electrical and Computer Engineering, University of California Santa Barbara, CA 93106. K. Fregene is with Lockheed Martin Advanced Technology Labs, Cherry Hill, NJ. B. M. Sadler is with Army Research Laboratory, Adelphi, MD 20783. Email: \{sriram, jtisaacs, hespanha, madhow $\} @$ ece.ucsb.edu, kingsley.fregene@lmco.com and brian.m.sadler6.civ@mail.mil.

This work was supported by the Institute for Collaborative Biotechnologies through grant W911NF-09-0001 from the U.S. Army Research Office. The content of the information does not necessarily reflect the position or the policy of the Government, and no official endorsement should be inferred. build a system that is very efficient in terms of minimizing the distance traversed while localizing the source.

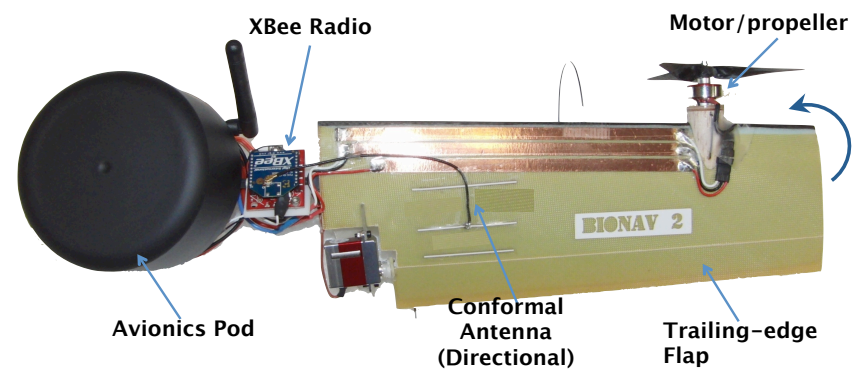

Fig. 1. A photograph of the MAV fitted with a conformal antenna and an Xbee radio. As the antenna rotates, the MAV estimates the source bearing to be the direction in which the RSS is largest and moves in this direction.

We use the single-wing rotating Samarai MAV presented in [1]. It consists of a pod-like structure that houses the electronics (including the microprocessor) and a rotating blade that is fitted to the avionics pod as shown in Figure 1. This design, inspired by the structure of maple seeds, has numerous mechanical advantages: it is simple, it is stable in hover, it has clean aerodynamics and it has a factor of ten smaller wing loading than conventional designs, which significantly reduces its power requirement. We attach a directional RF antenna to the rotating blade, which is key towards solving the source-seeking problem with low complexity: as the antenna rotates, we can measure the signal power incident from different directions. In a Line-of-Sight (LoS) scenario, the direction in which the RSS is maximal provides the bearing to the source. We build on this idea in choosing the MAVs path: after each rotation of the MAV, we average the angular RSS pattern observed over the previous few rotations and pick the direction in which the average RSS is largest. The MAV translates in this direction even as it continues to rotate and the process is repeated at the end of the next rotation. There is no mobility cost involved in making these bearing measurements, which is not the case for platforms that are not constantly rotating (such as fixed wing aircraft or ground robots), where a rotation of the platform necessarily slows down the translational motion. In our case, the singlewing MAV rotates whether it is translating or hovering and, therefore, the bearing information is always available. This scheme is also easy to implement: all that is needed for sensing is a directional antenna and a magnetometer (to establish a reference direction) and, in terms of storage/computation we only need to compute a running average of angular RSS 
patterns and choose the best direction.

While the simplicity of the proposed scheme makes it attractive, we also need to demonstrate that it is effective in realistic settings when the received signal includes interference from reflected paths in addition to the Line-of-Sight path. To provide some analytical insight into this question, we consider a simple model where each bearing estimate is either (a) the true bearing plus some relatively small additive noise, or (b) a complete outlier, perhaps originating off some reflectors whose positions we do not explicitly model. The latter occurs with some "outlier" probability $p$, while the former occurs with probability $1-p$. We prove convergence results for this model under two different, but both relatively weak, assumptions on the sequence of outlier values that we observe, thereby establishing the robustness of the algorithm. We then present simulations to quantify the performance of the proposed algorithm and experimental results that validate the theory and the simulations.

Contributions: Our main contributions can be summarized as follows:

- We propose a simple architecture for localizing an RF source using bearing measurements from a directional antenna mounted on a rotating MAV.

- We introduce an RF seeking algorithm that consists of three steps: (i) form the spatial RSS pattern observed after each rotation, (ii) reduce noise by passing the spatial RSS profile through an autoregressive filter and (iii) instruct the MAV to translate in the direction for which the averaged RSS pattern is largest.

- We prove two convergence results for a stochastic model of the proposed source-seeking algorithm: We first show that when the sequence of values taken by the outlier angles is independent and identically distributed (and extremely weak conditions that quantify how "small" the additive noise can be), the MAV will return to a small ball surrounding the source infinitely often. When the outlier bearings originate off a few fixed reflectors, assuming the outlier values to be independent might be optimistic. To avoid this assumption, we prove a similar result under more relaxed criteria on the sequence of outliers, but stronger assumptions on the probability with which they occur. Specifically, we show that if the outlier probability $p$ is smaller than a threshold depending only on the additive noise distribution, then no matter how the outlier values arise, the MAV will return to a small ball surrounding the source infinitely often.

- We present detailed Monte Carlo simulations to quantify the excess distance traversed by the MAV relative to the straight-line distance between its starting location and the source. Under the independent outlier model, we find that even for relatively large values of the outlier probability $p$ and additive noise, the excess distance traversed is small.

- We present experimental results where an MAV seeks an RF source starting from a distance of about $50 \mathrm{~m}$. While the algorithm is designed with stationary sources in mind, we show through our experiments that it is equally effective in following mobile sources.

Related Work: Source-seeking and localization of RF emit- ters has been a topic of extensive recent research. Much of the relevant related work falls into one or more of three categories, source-seeking, network localization, and bearing estimation.

Source-seeking with mobile agents has been studied for a number of fairly general signal fields and vehicle models [25]. While the concepts proposed in some of these references can be applied to the specific problem of seeking an RF source, we find that exploiting the properties of the RF field and the sensing architecture gives us substantial benefits. Closer to our work, in [6], the motion of a mobile robot is utilized along with an omni-directional radio to estimate the RSS gradient by taking signal strength measurements at a sequence of locations. This method depends on the mobile robot to accurately know its position relative to the starting point of the gradient measurement and also relies on monotonicity and symmetry of the signal strength decay as a function of distance between transmitter and receiver which may be problematic under multi-path scenarios. The problems of frontier exploration and radio source-seeking were addressed in [7] by using local RSS gradient estimates to govern which frontier waypoint to approach. In [8], a rotating directional antenna is mounted on a wheeled mobile robot for the purpose of wireless node localization. The RSS is measured as a function of antenna angle and crosscorrelation with a known antenna gain pattern is used to determine the relative bearing between an unknown radio source and the mobile robot. A particle filter is used to determine the location of the unknown radio source. Unlike our work, [8] needs to attach a servo motor for the robot to rotate, leading to extra payload and power requirements. A different approach to source-seeking that uses RSS measurements from an omnidirectional antenna mounted on a UAV to compute the spatial gradient is described in [9].

The fundamental problem of bearing estimation has received a lot attention. For example, a direction-of-arrival (DOA) sensor constructed using an actuated parabolic reflector is described in [10]. The DOA is found by identifying the direction of the maximum received signal strength indicator. In [11], the authors make the clever observation that while rotating a wireless antenna around a signal-blocking obstacle one can infer the angle of arrival of the received signal from the direction in which the signal was most attenuated.

In both [12] and [13], a mobile robot is equipped with a directional antenna for the purpose of localizing multiple stationary nodes in a wireless network. A localization algorithm based on a particle filter was used in [12], while a spatiotemporal probability occupancy grid was used in [13] to track posterior probability distributions of source locations. A Multi-Hypothesis Extended Kalman Filter framework is used to localize agents in a mobile robot team in [14], where each robot is equipped with an antenna that provides a $\pi$ periodic estimate of the relative bearing between each pair of communicating agents. 


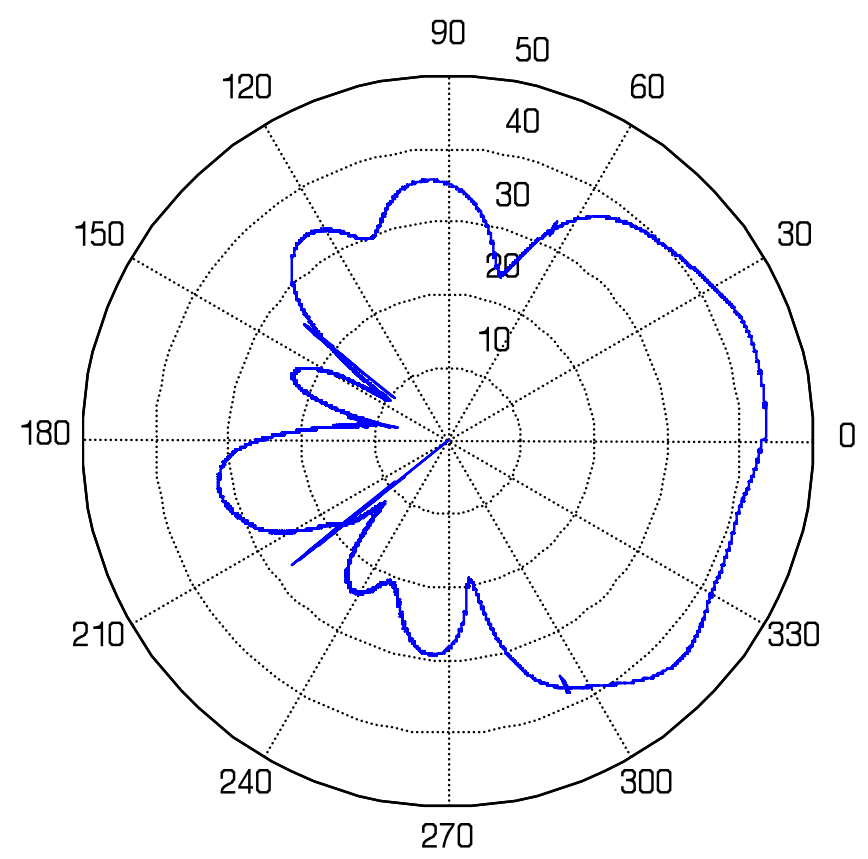

Fig. 2. The gain pattern of the directional antenna fitted on the rotating wing of the MAV.

\section{RSS MEASurements \& Algorithm}

The MAV is fitted with an XBee radio with a custom designed directional antenna whose gain pattern in the azimuthal plane is shown in Figure 2. We use the antenna to make RSS measurements 600 times a second as the MAV rotates and translates. We denote the RSS measurement at time $t$ by $P_{t}$. At the same time $t$, we use a magnetometer to estimate the antenna's orientation relative to a reference direction and denote it by $\beta_{t}$.

In a single path Line-of-Sight environment, the bearing to the source is given by the direction in which the RSS is largest as the MAV rotates. While this observation forms the basis of our algorithm, we need to modify it to better handle practical settings, where the received signal may have significant measurement noise, leading to large errors. To reduce such errors, we average the variations in the RSS patterns over multiple rotations.

Our implementation is further constrained by the fact that RSS patterns from different rotations can only be averaged in a coarse manner. As the MAV rotates, it only makes RSS measurements at discrete antenna orientations (say, spaced $7^{\circ}$ apart) and are not necessarily integer divisors of $360^{\circ}$. Therefore, the angles at which the RSS is measured varies across multiple rotations. Consequently, we coarsely average the RSS pattern over multiple rotations as follows: we split the angular space into appropriately sized bins so that, in every rotation, there is at least one RSS measurement in each bin. We use all the measurements that fall into a bin over a rotation to update the average RSS value corresponding to that bin.

Specifically, our algorithm proceeds in the following manner:
1) We divide the angular space into $B$ bins, with the $b$ th bin spanning $\left[\frac{2 \pi}{B}(b-1), \frac{2 \pi}{B} b\right], b=1,2, \ldots, B$.

2) We maintain an average RSS value for each bin and denote the value in the $b$ th bin by $Q[b]$.

3) As the MAV rotates, suppose that we get an RSS measurement $P_{t}$ and the corresponding yaw for the MAV $\beta_{t}$. We identify the bin in which the yaw falls (say bin $b_{0}$ ) and update the average RSS in this bin using an autoregressive filter:

$$
Q\left[b_{0}\right] \leftarrow \alpha Q\left[b_{0}\right]+(1-\alpha) P_{t} .
$$

4) At the end of a rotation, we pick the bin that has the largest value of the average RSS $Q[b]$. Denoting this bin by $\hat{b}$, we instruct the MAV to fly in a direction that makes an angle of $2 \pi \hat{b} / B$ with the azimuth.

5) We return to Step 3 and repeat the process.

In the rest of the paper, we assume that the bin size $B$ is given. A detailed analysis of the impact of the bin size is left for future work.

\section{ANALYSIS}

We now provide analytical insight into the algorithm using a stochastic model for the bearing measurements made after each rotation and proving that, under this model, the proposed algorithm will converge.

The bearing estimates typically fall into one of two categories: (a) they are roughly correct, with small errors that can be attributed to the bin quantization and small measurement noise or (b) they are outliers and have large errors, either due to large measurement noise or because signals from reflected paths distort the bearing estimate. We propose a first-order model to capture these possibilities: we assume that reasonably correct estimates occur with probability $1-p$ and outlier estimates occur with probability $p$. We analyze the algorithm under two different models for the sequence of outliers. First, we consider outlier values that are independent of one another, potentially caused by measurement noise that is occasionally large. Subsequently, we analyze the case where the outlier values are arbitrary and possibly highly correlated. This could arise from outlier estimates caused by reflectors, which typically would not be independent of each other. In both cases, we use recently established Lyapunov results to prove the recurrence of appropriately defined sets for discrete-time differential inclusions.

\section{A. Stochastic Motion Model}

Suppose that, after the $k$ th rotation, the MAV makes a bearing estimate $\psi_{k}$ of an RF source according to the following model:

$$
\psi_{k}=\left(1-\sigma_{k}\right)\left(\theta_{k}+n_{k}\right)+\sigma_{k} w_{k}, \quad \forall k \in \mathbb{Z}_{\geq 0},
$$

where $\theta_{k}$ denotes the true direction of the source after the $k$ th rotation, $n_{k}$ an i.i.d. sequence of additive noise, $w_{k}$ is a sequence of outlier measurements (not necessarily independent), and $\sigma_{k} \in\{0,1\}$ an i.i.d. sequence of Bernoulli random variables with parameter $p \in[0,1)$. The event $\sigma_{k}=1$ corresponds to the sensor producing an outlier 
measurement after the $k$ th rotation, that is unrelated to the true direction of the source.

Assuming the source is at the origin and the MAV is at $\left(x_{k}, y_{k}\right)$, the true source direction $\theta_{k}$ satisfies

$$
\cos \theta_{k}=-\frac{x_{k}}{r_{k}}, \quad \sin \theta_{k}=-\frac{y_{k}}{r_{k}} .
$$

where $r_{k}:=\sqrt{x_{k}^{2}+y_{k}^{2}}$ is the MAV's distance from the source.

We assume that at each time step, the MAV moves in the estimated source direction $\psi_{k}$ for a fixed distance $h$. Thus, the MAV's position satisfies the recursive relationship

$$
\left[\begin{array}{l}
x_{k+1} \\
y_{k+1}
\end{array}\right]=\left[\begin{array}{l}
x_{k} \\
y_{k}
\end{array}\right]+h\left[\begin{array}{c}
\cos \psi_{k} \\
\sin \psi_{k}
\end{array}\right]
$$

Using the trigonometric expansions

$$
\begin{aligned}
& \cos \left(\theta_{k}+n_{k}\right)=-\frac{x_{k} \cos n_{k}}{r_{k}}+\frac{y_{k} \sin n_{k}}{r_{k}}, \\
& \sin \left(\theta_{k}+n_{k}\right)=-\frac{x_{k} \sin n_{k}}{r_{k}}-\frac{y_{k} \cos n_{k}}{r_{k}},
\end{aligned}
$$

in (3), we conclude that

$$
\begin{gathered}
{\left[\begin{array}{l}
x_{k+1} \\
y_{k+1}
\end{array}\right]=\left(1-\sigma_{k}\right)\left[\begin{array}{c}
\left(1-h r_{k}^{-1} \cos n_{k}\right) x_{k}+h y_{k} r_{k}^{-1} \sin n_{k} \\
\left(1-h r_{k}^{-1} \cos n_{k}\right) y_{k}-h x_{k} r_{k}^{-1} \sin n_{k}
\end{array}\right]+} \\
\sigma_{k}\left[\begin{array}{c}
x_{k}+h \cos w_{k} \\
y_{k}+h \sin w_{k}
\end{array}\right] .
\end{gathered}
$$

\section{B. Stochastic Outliers}

We begin by considering the case where the sequence of outlier measurements $w_{k}$ are i.i.d.

Lemma 1: Assume that $n_{k}, w_{k}$, and $\sigma_{k}$ are i.i.d. sequences with

$$
\mathbb{E}\left[\cos w_{k}\right]=\mathbb{E}\left[\sin w_{k}\right]=0
$$

and that

$$
\eta:=\mathbb{E}\left[\cos n_{k}\right]>0
$$

Then, the set

$$
\mathcal{O}:=\left\{(x, y) \in \mathbb{R}^{2}: \sqrt{x^{2}+y^{2}}<\frac{h}{2(1-p) \eta}\right\}
$$

is globally recurrent for (3).

Before proving this result, it is worth emphasizing that this result holds under fairly mild assumptions: The condition (5) essentially requires the additive noise angle $n_{k}$ to be mostly concentrated in the region $[-\pi / 2, \pi / 2]$ for which the cosine is positive. The condition (4) requires some symmetry in the distribution of the outlier measurements so that the average of its sine and cosine are both zero.
Proof of Lemma 1. Defining $V(x, y):=\alpha\left(x^{2}+y^{2}\right)$, we have

$$
\begin{aligned}
& \mathbb{E}\left[V\left(x_{k+1}, y_{k+1}\right)-V\left(x_{k}, y_{k}\right) \mid x_{k}, y_{k}\right] \\
&=\alpha \mathbb{E}\left[\left(1-\sigma_{k}\right)\left(\left(1-h r_{k}^{-1} \cos n_{k}\right)^{2} r_{k}^{2}+h^{2} \sin ^{2} n_{k}\right)+\right.\left.\sigma_{k}\left(r_{k}^{2}+h^{2}+2 h x_{k} \cos w_{k}+2 h y_{k} \sin w_{k}\right)-r_{k}^{2} \mid x_{k}, y_{k}\right] \\
&=\alpha \mathbb{E}\left[\left(1-\sigma_{k}\right)\left(1+h^{2} r_{k}^{-2} \cos ^{2} n_{k}-2 h r_{k}^{-1} \cos n_{k}\right) r_{k}^{2}+\right. \sigma_{k}\left(h^{2}+2 h x_{k} \cos w_{k}+2 h y_{k} \sin w_{k}\right)+\left(\sigma_{k}-1\right) r_{k}^{2}+ \\
&\left.\left(1-\sigma_{k}\right) h^{2} \sin ^{2} n_{k} \mid x_{k}, y_{k}\right] \\
&=\alpha \mathbb{E}\left[h^{2}-2 h\left(1-\sigma_{k}\right) r_{k} \cos n_{k}+2 h \sigma_{k} x_{k} \cos w_{k}+\right. \\
&\left.\quad 2 h \sigma_{k} y_{k} \sin w_{k} \mid x_{k}, y_{k}\right] .
\end{aligned}
$$

Using the fact that $n_{k}, w_{k}$, and $\sigma_{k}$ are i.i.d., we conclude that

$$
\begin{gathered}
\mathbb{E}\left[V\left(x_{k+1}, y_{k+1}\right)-V\left(x_{k}, y_{k}\right) \mid x_{k}, y_{k}\right] \\
=\alpha h^{2}+2 \alpha h p\left(x_{k} \mathbb{E}\left[\cos w_{k}\right]+y_{k} \mathbb{E}\left[\sin w_{k}\right]\right) \\
\quad-2 \alpha h(1-p) \mathbb{E}\left[\cos n_{k}\right] r_{k} \\
=-2 \alpha h(1-p) \eta r_{k}+\alpha h^{2}
\end{gathered}
$$

where we used (4) in the second equality. Setting $\alpha:=1 / h^{2}$, we have

$$
\begin{aligned}
& \mathbb{E}\left[V\left(x_{k+1}, y_{k+1}\right)-V\left(x_{k}, r_{k}\right) \mid x_{k}, y_{k}\right] \\
& =-2 h^{-1}(1-p) \eta r_{k}+1=-\rho\left(r_{k}\right)+I_{<\epsilon}\left(r_{k}\right),
\end{aligned}
$$

where

$$
\rho(r)= \begin{cases}2 h^{-1}(1-p) \eta r & r<\epsilon \\ 2 h^{-1}(1-p) \eta r-1 & r \geq \epsilon\end{cases}
$$

and $I_{<\epsilon}(r)$ is the indicator function taking the value 1 when $r<\epsilon$ and 0 otherwise. Assuming that $\eta>0$, this function is positive as long as

$$
2 h^{-1}(1-p) \eta \epsilon>1 \Leftrightarrow \epsilon>\frac{h}{2(1-p) \eta},
$$

from which we can conclude that the following set is globally recurrent

$$
\mathcal{O}:=\left\{(x, y) \in \mathbb{R}^{2}: \sqrt{x^{2}+y^{2}}<\frac{h}{2(1-p) \eta}\right\}
$$

[15], i.e., the state $\left(x_{k}, r_{k}\right)$ will always return to this set with probability one.

\section{Worst-case outliers}

When the outliers result from reflections off a specific object, the sequence of outliers $w_{k}$ need not be i.i.d. To account for such scenarios, we allow for any arbitrary (worstcase) sequence of outliers $w_{k} \in[0,2 \pi]$.

Lemma 2: Assume that $n_{k}$ and $\sigma_{k}$ are i.i.d. sequences and that

$$
p<\frac{\eta}{\sqrt{2}+\eta}, \quad \eta:=\mathbb{E}\left[\cos n_{k}\right]>0 .
$$


Then, the set

$$
\mathcal{O}:=\left\{(x, y) \in \mathbb{R}^{2}: \sqrt{x^{2}+y^{2}}<\frac{h}{2(\eta-p \eta-p \sqrt{2})}\right\}
$$

is globally recurrent for any solution to (3) that is adapted to the filtration generated by $\left(n_{k}, \sigma_{k}\right), k \in \mathbb{Z}_{\geq 0}$ with $w_{k} \in$ $[0,2 \pi]$.

We emphasize that Lemma 2 no longer places any assumption on the sequence of outliers. The price to pay is that we can only guarantee convergence when the probability of an outlier measurement satisfies (6). Lemma 2 applies to any solution to (3) that is adapted to the filtration generated by $\left(n_{k}, \sigma_{k}\right), k \in \mathbb{Z}_{\geq 0}$. This technical condition is needed when one mixes worst-case selections with stochastic processes and it essentially excludes the possibility for selections of a worst-case outlier $w_{k}$ that depends on future realizations of the stochastic processes $\eta_{\ell}, \sigma_{\ell}$, for $\ell>k$ [15].

Proof of Lemma 2. As in the proof of Lemma 1, we find a globally recurrent set by computing

$$
\begin{aligned}
& \mathbb{E}\left[\sup _{w_{k}} V\left(x_{k+1}, y_{k+1}\right)-V\left(x_{k}, y_{k}\right) \mid x_{k}, y_{k}\right] \\
&=\alpha \mathbb{E}\left[\sup _{w_{k}} h^{2}-2 h\left(1-\sigma_{k}\right) r_{k} \cos n_{k}+2 h \sigma_{k} x_{k} \cos w_{k}+\right. \\
&\left.2 h \sigma_{k} y_{k} \sin w_{k} \mid x_{k}, y_{k}\right] \\
&= \alpha \mathbb{E}\left[h^{2}-2 h\left(1-\sigma_{k}\right) r_{k} \cos n_{k}+2 h \sigma_{k}\left(\left|x_{k}\right|+\left|y_{k}\right|\right) \mid x_{k}, y_{k}\right] \\
&= \alpha h^{2}-2 \alpha h(1-p) \eta r_{k}+2 \alpha h p\left(\left|x_{k}\right|+\left|y_{k}\right|\right) .
\end{aligned}
$$

Using the fact that

$$
\left|x_{k}\right|+\left|y_{k}\right| \leq \sqrt{2} r_{k}
$$

we conclude that

$$
\begin{array}{r}
\mathbb{E}\left[\sup _{w_{k}} V\left(x_{k+1}, y_{k+1}\right)-V\left(x_{k}, y_{k}\right) \mid x_{k}, y_{k}\right] \\
\leq-2 \alpha h((1-p) \eta-p \sqrt{2}) r_{k}+\alpha h^{2} .
\end{array}
$$

Setting $\alpha:=1 / h^{2}$, we have

$$
\begin{aligned}
& \mathbb{E}\left[V\left(x_{k+1}, y_{k+1}\right)-V\left(x_{k}, r_{k}\right) \mid x_{k}, y_{k}\right] \\
& \leq-2 h^{-1}((1-p) \eta-p \sqrt{2}) r_{k}+1=-\rho\left(r_{k}\right)+I_{<\epsilon}\left(r_{k}\right),
\end{aligned}
$$

where

$$
\rho(r):= \begin{cases}2 h^{-1}((1-p) \eta-p \sqrt{2}) r & r<\epsilon \\ 2 h^{-1}((1-p) \eta-p \sqrt{2}) r-1 & r \geq \epsilon .\end{cases}
$$

Assuming that

$$
(1-p) \eta-p \sqrt{2}>0 \Leftrightarrow p<\frac{\eta}{\sqrt{2}+\eta},
$$

this function is positive as long as

$$
2 h^{-1}((1-p) \eta-p \sqrt{2}) \epsilon>1 \Leftrightarrow \epsilon>\frac{h}{2(\eta-p \eta-p \sqrt{2})},
$$

from which we can conclude that the following set is globally recurrent

$$
\mathcal{O}:=\left\{(x, y) \in \mathbb{R}^{2}: \sqrt{x^{2}+y^{2}}<\frac{h}{2(\eta-p \eta-p \sqrt{2})}\right\}
$$

[15], i.e., the state $\left(x_{k}, r_{k}\right)$ will always return to this set with probability one.

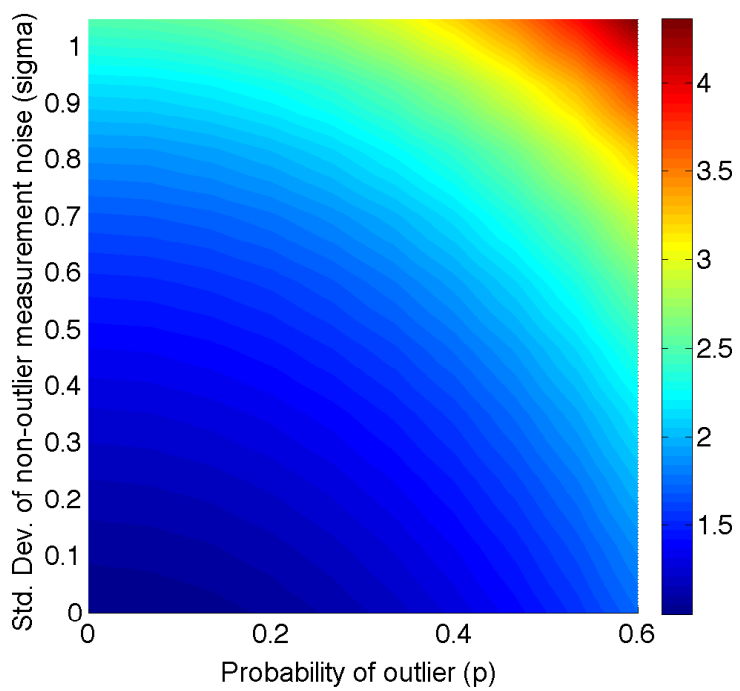

Fig. 3. Average stretch for different choices of outlier probability $p$ and standard deviation of additive noise $\sigma$.

\section{Simulation Results}

To simulate the algorithm, we begin with the RF source at the origin of a two-dimensional plane and the MAV at position $(50 \mathrm{~m}, 50 \mathrm{~m})$. At each time step, the MAV makes a bearing measurement according to the model in (2). The additive noise $n_{k}$ and the outliers $w_{k}$ are both independent across time with $n_{k} \sim N\left(0, \sigma^{2}\right)$ and $w_{k}$ is chosen uniformly from $[0,2 \pi]$. The MAV then moves a distance $h=0.1 \mathrm{~m}$ in the direction of the bearing, after which the process is repeated. We terminate the simulation when the MAV is within $1 \mathrm{~m}$ of the source. We run simulations for multiple values of the outlier probability $p$ between 0 and 0.6 and the standard deviation of the additive noise $\sigma$ between 0 and $\pi / 3$. For each choice of $p$ and $\sigma$, we run 1200 trials. We quantify the algorithm's performance by its stretch, defined to be the ratio of the distance traversed by MAV and the straight-line distance between the source and the MAV's starting location.

We plot the average stretch for different choices of $p$ and $\sigma$ in Figure 3. We observe that even for relatively large values of $p$ and $\sigma$, the stretch is fairly small. For example, the stretch is only 1.6 even when outliers occur with a probability $p=$ 0.4 and the noise standard deviation $\sigma=20^{\circ}$.

We plot the distribution of the stretch in Figure 4 to understand the variability in performance. Specifically, we plot histograms of the stretch for different values of $\sigma$, with $p$ set to 0.2667 in Figure 4(a). Similarly, Figure 4(b) plots histograms of the stretch for different choices of $p$ with $\sigma=0.4654$. We see from this figure that the distribution in the stretch increases fairly fast with the probability of outliers. On the other hand, Figure 4(a) shows that the distribution in the stretch increases much more slowly with the standard deviation of the additive noise $\sigma$. The reason for this is intuitive: additive noise perturbs the true estimate, but the MAV still moves in the general direction of the source. Outliers, however, can send the MAV in any direction. Thus, 

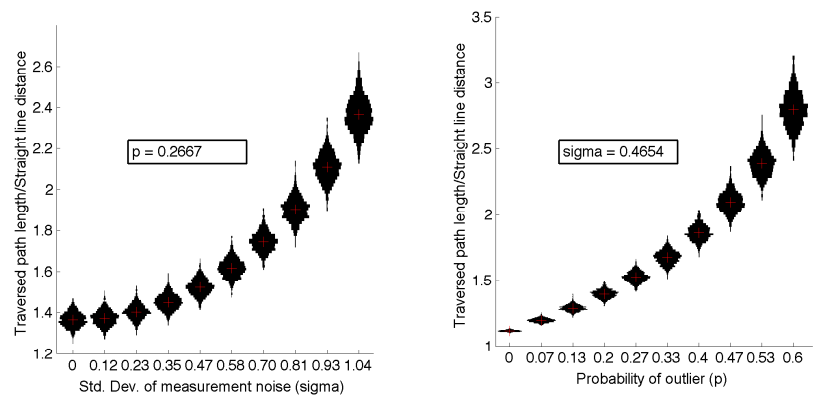

(a) Distribution of stretch for differ- (b) Distribution of the stretch for difent values of $\sigma$, with $p$ set to 0.2667 . ferent values of $p$, with $\sigma$ set to 0.4654 .

Fig. 4. Distribution of the stretch as we vary one of the two parameters $p$ and $\sigma$ while holding the other fixed.

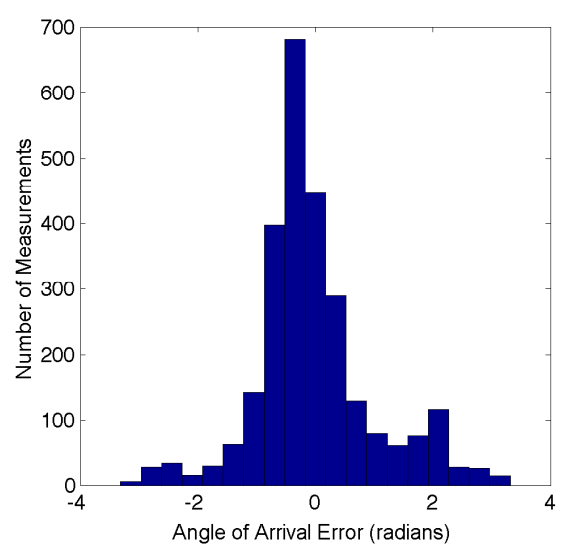

Fig. 5. Distribution of Angle of Arrival errors.

as the probability of outliers increase, the MAV makes random-walk like steps more frequently, thereby increasing the variability in the stretch.

\section{EXPERIMENTAL RESULTS}

\section{A. Angle of Arrival Verification Test}

The first experiment was designed to characterize the bearing errors seen by the MAV as well as the probability of an outlier. The experiments were conducted in a domed soccer field complex. We instructed the MAV to fly in a crisscross pattern around a stationary source while recording the AOA estimate, GPS location of the source, GPS location of the MAV, and raw RSS. The histogram of the error between the estimated AOA and ground truth AOA calculated from comparing GPS measurements of the MAV and source can be seen in Figure 5. Out of 2671 AOA estimates there were 613 errors of greater than $\pi / 3$, and the mean and standard deviation of all 2671 measurements were -0.04 and 1.02 radians, respectively.

\section{B. Stationary Source-Seeking and Mobile Source-Tracking}

We ran several experiments using the algorithm described in Section II with the shortest distance between the MAV's starting position and the source at roughly 50 meters. In the example run shown in Figure 6, the MAV started at a distance

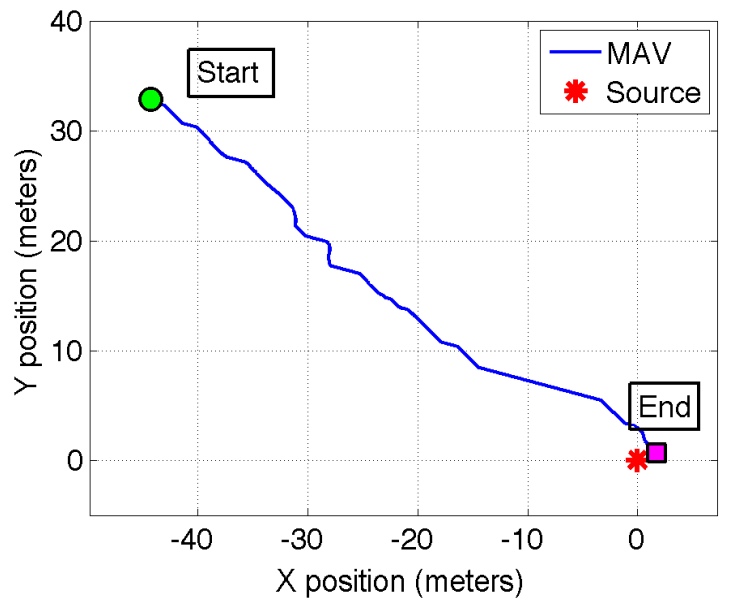

(a) MAV's trajectory as it seeks a stationary source located at the origin.

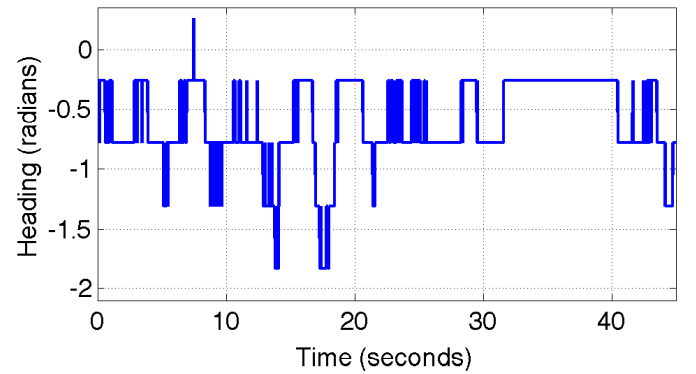

(b) Headings computed by MAV after each rotation

Fig. 6. Results from MAV seeking a stationary source showing the MAV's trajectory and the headings that it follows.

of 55.16 meters and covered distance of 59.85 meters to find the source. The sequence of bearing estimates computed using the source-seeking algorithm is plotted in Figure 6(b).

In addition to the successful experiments with a stationary source, we have also found in preliminary tests that the greedy algorithm is successful in tracking a moving source. An example run is shown in Figure 7. The source (red trace) starts at $[7,-23]$ while the MAV (blue trace) starts at $[6,-14]$. As the MAV gets close to the source, the source moves in the positive $\mathrm{Y}$ direction. Then the source moves to $[-9,-4]$, the MAV follows, overshoots the source location slightly, and returns towards the source. The MAV repeats this behavior as the source moves. We also plot the sequence of bearing estimates computed using the sourceseeking algorithm in Figure 7(b).

\section{Conclusions}

We have proposed and demonstrated a minimalist architecture for RF source-seeking using a lightweight rotating MAV. The key idea is to derive bearing estimates using a directional antenna on the MAV's rotating blade. Greedy translation in the direction of these bearing estimates is proven to converge even in the presence of outliers which are not necessarily independent, and the dependence of stretch (relative to the shortest path) on the statistics of the measurement noise and outliers is quantified via simulations. Indoor experiments 


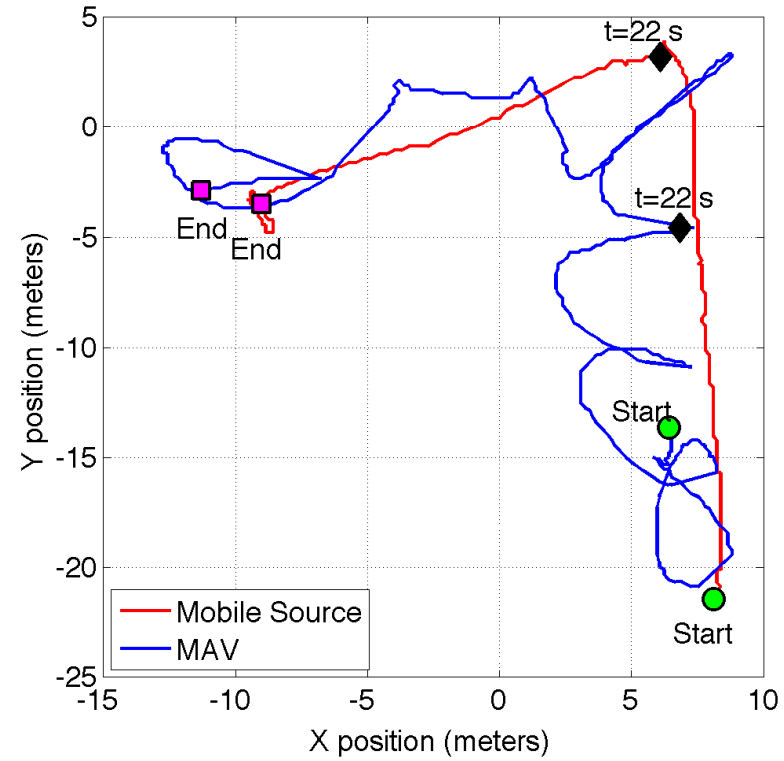

(a) MAV's trajectory as it tracks a moving source

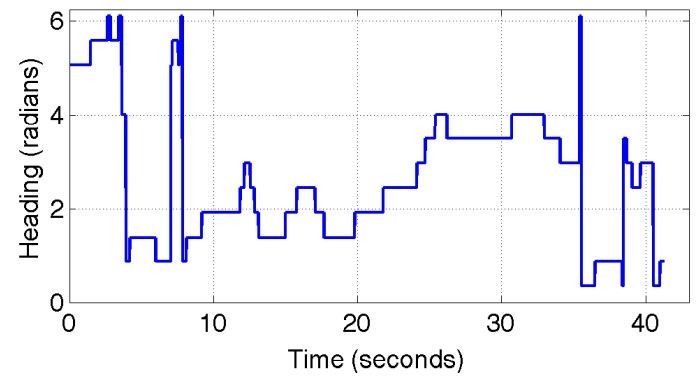

(b) Headings computed by MAV after each rotation

Fig. 7. Results from MAV tracking a moving source showing the MAV's trajectory and the headings that it follows.

demonstrate the performance of the algorithm both for static and moving sources. Important topics for future work include refinement of our analytical model based on electromagnetic propagation modeling, extending source seeking to three dimensions by electrically steering the antenna pattern or mechanically orienting the MAV, extension of our analytical framework to mobile sources and realistic MAV motion models, and detailed experimental validation in both indoor and outdoor environments.

\section{ACKNOWLEDGMENT}

We would like to thank Aseem Wadhwa at UCSB for helping with an earlier version of the experiments.

\section{REFERENCES}

[1] K. Fregene and C. Bolden, "Dynamics and control of a biomimetic single-wing nano air vehicle," in American Control Conference, Baltimore, MD, June 2010, pp. $51-56$.

[2] C. Mayhew, R. Sanfelice, and A. Teel, "Robust sourceseeking hybrid controllers for autonomous vehicles," in American Control Conference, New York, NY, July 2007, pp. 1185-1190.
[3] A. R. Mesquita, J. P. Hespanha, and K. Åström, "Optimotaxis: A stochastic multi-agent on site optimization procedure," in Hybrid Systems: Computation and Control, ser. Lecture Notes in Computer Science, M. Egerstedt and B. Mishra, Eds. Springer-Verlag, 2008, no. 4981, pp. 358-371.

[4] S.-J. Liu and M. Krstic, "Stochastic nonholonomic source seeking," in IEEE Conference on Decision and Control, Atlanta, GA, December 2010, pp. 6985-6990.

[5] S. Azuma, M. Sakar, and G. Pappas, "Nonholonomic source seeking in switching random fields," in IEEE Conference on Decision and Control, Atlanta, GA, December 2010, pp. 6337-6342.

[6] K. Dantu, P. Goyal, and G. S. Sukhatme, "Relative bearing estimation from commodity radios," in IEEE International Conference on Robotics and Automation, Kobe, Japan, May 2009, pp. 3215-3221.

[7] J. Twigg, J. Fink, P. Yu, and B. Sadler, "RSS gradientassisted frontier exploration and radio source localization," in International Conference on Robotics and Automation, St. Paul, MN, May 2012, pp. 889-895.

[8] J. Graefenstein, A. Albert, P. Biber, and A. Schilling, "Wireless node localization based on RSSI using a rotating antenna on a mobile robot," in Workshop on Positioning, Navigation and Communication, Hanover, Germany, March 2009, pp. 253-259.

[9] A. Wadhwa, U. Madhow, J. Hespanha, and B. Sadler, "Following an RF trail to its source," in 49th Annual Allerton Conference on Communication, Control and Computing, Monticello, IL, October 2011, pp. 580-587.

[10] B. Hood and P. Barooah, "Estimating DoA from radiofrequency RSSI measurements using an actuated reflector," IEEE Sensors Journal, vol. 11, no. 2, pp. 413-417, 2011.

[11] Z. Zhang, X. Zhou, W. Zhang, Y. Zhang, G. Wang, B. Y. Zhao, and H. Zheng, "I am the antenna: accurate outdoor AP location using smartphones," in Conference on Mobile Computing and Networking, Las Vegas, NV, 2011, pp. 109-120.

[12] D. Song, J. Yi, and Z. Goodwin, "Localization of unknown networked radio sources using a mobile robot with a directional antenna," in American Control Conference, New York, NY, July 2007, pp. 5952-5957.

[13] D. Song, C.-Y. Kim, and J. Yi, "Monte Carlo simultaneous localization of multiple unknown transient radio sources using a mobile robot with a directional antenna," in International Conference on Robotics and Automation, Kobe, Japan, May 2009, pp. 3154-3159.

[14] J. Derenick, J. Fink, and V. Kumar, "Localization using ambiguous bearings from radio signal strength," in International Conference on Intelligent Robots and Systems, San Francisco, CA, Sept 2011, pp. 3248-3253.

[15] A. R. Teel, J. P. Hespanha, and A. Subbaraman, "Stochastic difference inclusions: results on recurrence and asymptotic stability in probability," in IEEE Conference on Decision and Control, Maui, HI, December 2012, to appear. 